\title{
Detecting Specific Oscillatory Modes in the Dynamics of Erbium-Doped Fiber Laser
}

\author{
M. O. Zhuravlev , A. A. Koronovskii ， O. I. Moskalenko , A. N. Pisarchik \\ D.-R. Rider , and A. E. Hramov
}

\begin{abstract}
A method for determining the oscillatory mode occurring in an erbium-doped fiber laser with a modulated parameter is proposed. The method is based on using a continuous wavelet transform with a mother Morlet wavelet and analyzing the energy of the wavelet spectrum that corresponds to the relevant range of time scales.
\end{abstract}

\section{INTRODUCTION}

One popular interdisciplinary trend is studying the nonlinear dynamics of fiber lasers, as is confirmed by the many works devoted to this topic [1-3]. Despite this, however, some problems in this domain remain to be solved. One such problem is studying the behavior of an erbium-doped fiber laser with a modulated parameter in which several oscillatory modes can exist simultaneously; these modes differ in the frequency and amplitude of the generated radiation [4]. Fluctuations can result in an originally multistable system being transformed into a metastable one, and in intermittent behavior that can be observed in the dynamics of the system (e.g., noise-induced switchings between different attractors). This changes the frequency and power of the laser radiation $[4,5]$. Because several modes coexist simultaneously, the observed switchings between the attractors cause intermittency of the laser that has not been classified to date, since the characteristics of the intermittent behavior correspond to none of the known types of intermittency. The problem of determining the type of intermittent behavior occurring in this system thus arises, since the expected results would help considerably in understanding the processes that lead to multistability in the erbium-doped fiber laser. This in turn would provide efficient tools for suppressing multistability in lasers of this type.

As a rule, determining the type of the intermittent behavior existing in the system under study requires that statistical characteristics of this behavior be found, especially the dependence of the average duration of laminar behavior on the parameter of supercriticality and the distribution of the durations of the laminar intervals of such behavior at constant control parameters. A major problem that arises in studying intermittency is therefore to detect time intervals in signals that correspond to different types of dynamics of systems with intermittent behavior; in practice, this is a problem of detecting laminar and turbulent phases. There are a number of methods that allow us to detect laminar intervals of the behavior in time functions; due to specific features of the system under consideration, however, none of the available methods allow us to solve the problem of detecting the characteristic intervals of the behavior occurring in an erbium-doped fiber laser with a modulated parameter. We must therefore develop a method that would enable us to detect the oscillatory mode in the system at a specific moment of time; this in turn will pave the way for determining the type of intermittency occurring in an erbium-doped fiber laser.

In this work, we propose a new method for detecting the characteristic oscillatory modes occurring in an erbium-doped fiber laser with a modulated parameter. The method is based on using a continuous wavelet transform with a mother Morlet wavelet [6] and analyzing the energy of the wavelet spectrum that corresponds to the relevant range of time scales [7, 8].

\section{CONTINUOUS WAVELET TRANSFORM}

The continuous wavelet transform with a complex mother wavelet for chaotic time series $x(t)$ can be written as [6]

$$
W\left(s, t_{0}\right)=\frac{1}{\sqrt{s}} \int_{-\infty}^{+\infty} x(t) \psi^{*}\left(\frac{t-t_{0}}{s}\right) d t,
$$




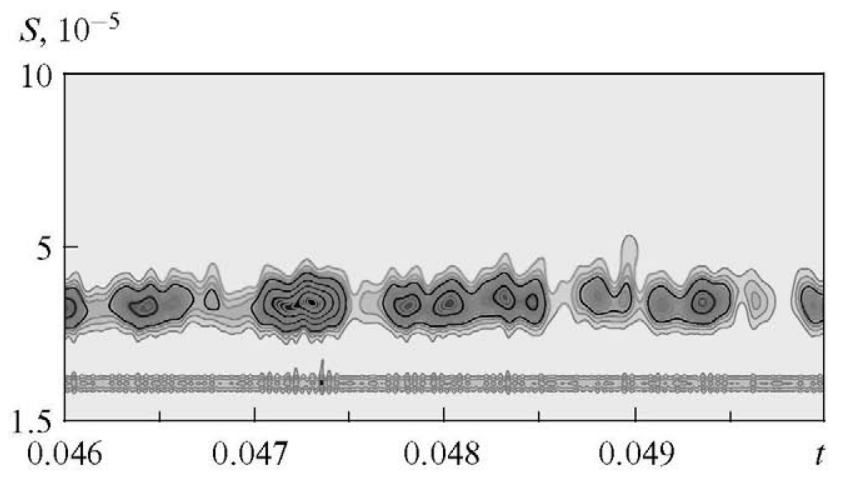

Fig. 1. Time dependence of the wavelet spectrum for an erbium-doped fiber laser with a modulated parameter. The modulated parameter is $P=13$.

where $x(t)$ is the time function of a chaotic signal; $\psi_{s, t_{0}}(t)$ is the mother wavelet; and $s$ is the time scale that determines the width of the wavelet; the asterisk denotes complex conjugation. We use the time dependence of the radiation intensity as signal $x(t)$ for the erbium-doped fiber laser under study.

As the mother wavelet, we use the complex Morlet wavelet [6]

$$
\psi(\eta)=(1 / \sqrt[4]{\pi}) \exp \left(j \Omega_{0} \eta\right) \exp \left(-\eta^{2} / 2\right),
$$

with parameter $\Omega_{0}=2 \pi$, which establishes a clear relationship between the time scale of wavelet transform $s$ and the frequency of Fourier transform $f: f=1 / s$.

Like the power spectrum of the Fourier transform, we can introduce [6] the distribution of the energy over the scales of wavelet transform

$$
E\left(s, t_{0}\right)=|W(s, t)|^{2}
$$

and integral time-averaged wavelet spectrum

$$
\langle E(s)\rangle=\int\left|W\left(s, t_{0}\right)\right|^{2} d t_{0} .
$$

Note that a distinctive feature of the continuous wavelet transform is that this method for analyzing compound chaotic signals enables us to detect the contribution from a particular time scale (frequency) at a specific moment in time; this in turn allows us to determine the oscillatory mode existing in the system at this moment in time.

\section{DETECTING DIFFERENT OSCILLATORY MODES}

In order to determine the oscillatory mode existing in an erbium-doped fiber laser with a modulated parameter at different moments in time, we used a method of detection that was based on analyzing the instantaneous distribution of the energy of a continuous wavelet transform on characteristic time scales.
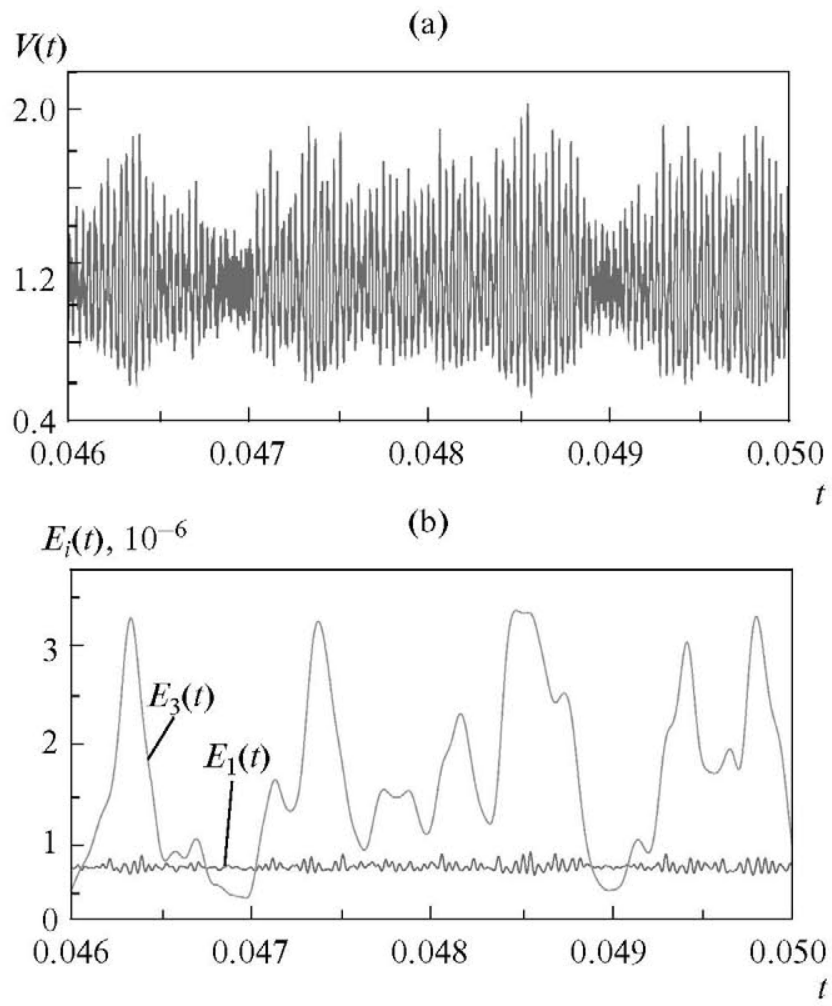

Fig. 2. (a) Time dependence of the radiation intensity for an erbium-doped fiber laser with modulated parameter $P=13$ and (b) normalized time dependences of the instantaneous distribution of the energy of the wavelet transform for period one with $f_{1}=80 \mathrm{kHz}\left(s_{1}=0.0000125\right)$ and period three with $f_{3}=26 \mathrm{kHz}\left(s_{3}=0.0000384\right)$.

Assessing the characteristic time scales in a signal under study requires the time dependence of the wavelet spectrum to be plotted. Note that this study was conducted for an erbium-doped fiber laser with a modulated parameter, and that the fundamental frequency of the intensity of oscillation was $f=80 \mathrm{kHz}$. Figure 1 shows the time dependence of the wavelet spectrum for an erbium-doped fiber laser with a modulated parameter (the modulation parameter was $P=13$ ). In the time dependence of the wavelet spectrum presented in Fig. 1, we can see that there are two characteristic periods of oscillations in the signal: period one with oscillation frequency $f_{1}=80 \mathrm{kHz}$ and period three with oscillation frequency $f_{3}=26 \mathrm{kHz}$. There is thus switching between two different oscillatory modes in the system. In order to determine which of the two modes exists in the system at the moment, we must compare the values of the instantaneous distributions of the energy of the wavelet transform on the characteristic time scales while allowing for the normalization of the wavelet spectrum. Figure $2 b$ shows the normalized time dependences of the instantaneous distribution of the energy of the wavelet transform for 


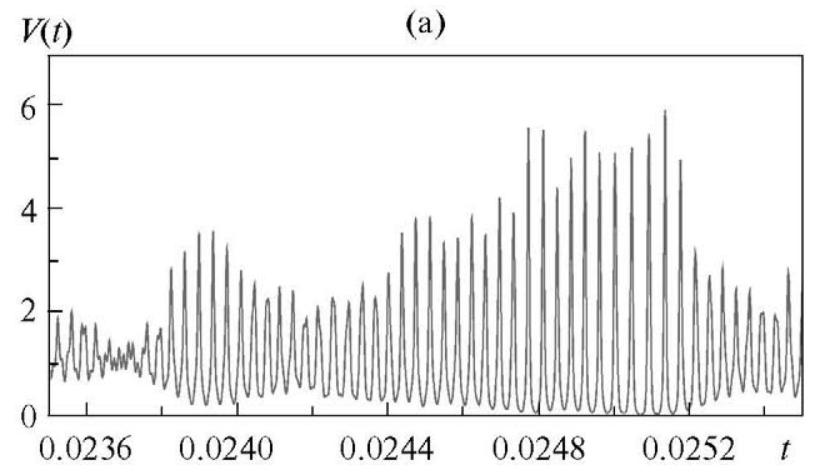

$E_{i}(t), 10^{-6}$

(b)

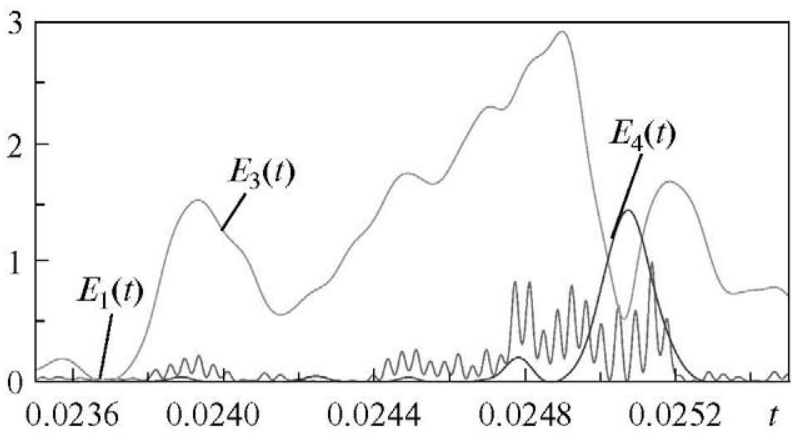

Fig. 3. (a) Time dependence of the radiation intensity for an erbium-doped fiber laser with modulated parameter $P=27$ and (b) normalized time dependences of the instantaneous distribution of energy of the wavelet transform for period one with $f_{1}=80 \mathrm{kHz}\left(s_{1}=0.0000125\right)$, period three with $f_{3}=26 \mathrm{kHz}\left(s_{3}=0.0000384\right)$, and period four with $f_{4}=20 \mathrm{kHz}\left(s_{4}=0.00005\right)$.

period one with $f_{1}=80 \mathrm{kHz}\left(s_{1}=0.0000125\right)$ and period three with $f_{3}=26 \mathrm{kHz}\left(s_{3}=0.0000384\right)$. Two characteristic regions can be distinguished in Fig. $2 \mathrm{~b}$ : region $\mathrm{I}$, in which the instantaneous distribution of the energy of the wavelet transform $E_{1}\left(s_{1}, t_{0}\right)$ has the maximum value for period one with $f_{1}$, and region $\mathrm{II}$, in which the instantaneous distribution of the energy of wavelet transform $E_{3}\left(s_{3}, t_{0}\right)$, which corresponds to period three with $f_{3}$, reaches its maximum value. Figure 2 a shows the time dependence of the radiation intensity for an erbium-doped fiber laser. In this figure too, we can see two characteristic regions in the behavior of the radiation intensity (periods one and three) whose time intervals completely agree with those shown in Fig. $2 b$. Our method based on analyzing the instantaneous distribution of the energy of the wavelet transform over time on characteristic time scales thus allows us to detect the oscillatory mode occurring in an erbiumdoped fiber laser with a modulated parameter at a specific moment in time.

Note that our method for detecting characteristic oscillatory modes of the behavior of an erbium-doped fiber laser also ensures its correct operation if more than two different oscillatory modes exist simultaneously in the system. Figure $3 \mathrm{~b}$ shows the normalized time dependences of the instantaneous distribution of the energy of the wavelet transform for period one with $f_{1}=80 \mathrm{kHz}\left(s_{1}=0.0000125\right)$, period three with $f_{3}=$ $26 \mathrm{kHz}\left(s_{3}=0.0000384\right)$, and period four with $f_{4}=$ $20 \mathrm{kHz}\left(s_{4}=0.00005\right)$; the modulation parameter for an erbium-doped fiber laser was $P=30$. Three characteristic regions can be distinguished in Fig. 3b: region I, in which the instantaneous distribution of the energy of the wavelet transform $E_{1}\left(s_{1}, t_{0}\right)$ has the maximum value for period one; region II, in which the instantaneous distribution of the energy of the wavelet transform $E_{3}\left(s_{3}, t_{0}\right)$, corresponding to period three has its maximum value; and region III where the instantaneous distribution of the energy of the wavelet transform $E_{4}\left(s_{4}, t_{0}\right)$ corresponding to period four has its maximum value. All of these regions are also presented in Fig. 3a, which shows the time dependences of the radiation intensity; it is clear that the radiation intensity displays specific behavior in each region, and that the mode of behavior changes at each interface of the regions.

\section{CONCLUSIONS}

A new method based on using the instantaneous distribution of the energy of a wavelet transform and the time dependence of a wavelet spectrum was proposed. Our method allows us to determine the oscillatory modes that can be observed for the radiation intensity of an erbium-doped fiber laser with a modulated parameter. In addition, it can be used to determine the moment in time at which the mode of oscillation changes; this in turn enables us to determine the duration of a specific period of oscillations in a system under study.

We may therefore assert that the proposed method for detecting characteristic oscillatory modes in an erbium-doped fiber laser with a modulated parameter will allow us to study intermittent behavior when there are two or more characteristic periods of oscillations. These studies will contribute to our understanding of the processes that lead to multistability in an erbiumdoped fiber laser; this in turn will provide more efficient tools for suppressing multistability in lasers of this type.

\section{ACKNOWLEDGMENTS}

This work was supported by the Russian Foundation for Basic Research, project no. 15-32-20299. 


\section{REFERENCES}

1. Huerta-Cuellar, G., Pisarchik, A.N., and Barmenkov, Y.O., Phys. Rev. E, 2008, vol. 78, p. 035202(R).

2. Huerta-Cuellar, G., Pisarchik, A.N., Kiryanov, A.V., Barmenkov, Yu.O., and del Valle Hernandez, J., Phys. Rev. E, 2009, vol. 79, p. 036204.

3. Pisarchik, A.N. and Jaimes-Reategui, R., Phys. Lett. A, 2009, vol. 374, p. 228.

4. Pisarchik, A.N., Jaimes-Reategui, R., Sevilla-Escoboza, R., and Huerta-Cuellar, G., Phys. Rev. E., 2012, vol. 86, p. 056219.
5. Pisarchik, A.N., Jaimes-Reategui, R., Sevilla-Escoboza, R., Huerta-Cuellar, G., and Taki, M., Phys. Rev. Lett., 2011, vol. 107, p. 274101.

6. Torresani, B., Continuous Wavelet Transform, Paris: Savoire, 1995.

7. Hramov, A.E., Koronovskii, A.A., Midzyanovskaya, I.S., and Sitnikova, E.Yu., Chaos, 2006, vol. 16, p. 043111.

8. Sitnikova, E.Yu., Hramov, A.E., Grubov, V.V., and Koronovskii, A.A., Brain Res., 2014, vol. 1543, p. 290.

Translated by D. Tkachuk 